\title{
Prevalence and Prognostic Factors of Disability After Major Trauma
}

\author{
Akkie N. Ringburg, MD, PhD, Suzanne Polinder, PhD, Marie Catherine P. van Ierland, MSc, \\ Ewout W. Steyerberg, PhD, Esther M. M. van Lieshout, PhD, Peter Patka, MD, PhD, \\ Ed F. van Beeck, $M D, P h D$, and Inger B. Schipper, $M D, P h D$
}

\begin{abstract}
Background: The primary aim of this study was to assess the health-related quality of life of survivors of severe trauma 1 year after injury, specified according to all the separate dimensions of the EuroQol-5D (EQ-5D) and the Health Utilities Index (HUI).

Methods: A prospective cohort study was conducted in which all severely injured trauma patients presented at a Level I trauma center were included. After 12 months, the EQ-5D, HUI2 and HUI3 were used to analyze the health status.

Results: Follow-up assessments were obtained from 246 patients (response rate, $68 \%$ ). The overall population EQ-5D (median) utility score was 0.73 (EQ-5D Dutch general population norm, 0.88). HUI2, HUI3, and EQ-5D Visual Analog Scale scores were 0.81, 0.65, and 70, respectively. Eighteen percent had at least one functional limitation 1 year after trauma, and $60 \%$ reported functional limitations on two or more domains using the EQ-5D. The female gender and comorbidity were significant independent predictors of disability.

Conclusion: Functional outcome and quality of life of survivors of severe injury have not returned to normal 1 year after trauma. The prevalence of specific limitations in this population is very high $(40-70 \%)$. Female gender and comorbidity are predictors of long-term disability.
\end{abstract}

Key Words: Health-related quality of life, Outcome studies, Diability, EQ-5D, HUI.

( Trauma. 2011;70: 916-922)

T raumatic injuries are the leading cause of death worldwide in persons younger than 45 years. ${ }^{1}$ Major trauma (defined as Injury Severity Score $[\mathrm{ISS}] \geq 16$ ) has such a large impact because of the relatively young age of the average severely injured patient. ${ }^{2}$

In addition to fatal outcomes, disabilities (i.e., reduced levels of functioning resulting from diseases or injuries ${ }^{3}$ ) are increasingly seen as an important component of a population's health. This has been recognized in the field of injury

Submitted for publication November 26, 2009.

Accepted for publication August 11, 2010.

Copyright (C) 2011 by Lippincott Williams \& Wilkins

From the Departments of Surgery-Traumatology (A.N.R., M.-C.P.v.I., E.M.M.v.L., P.P., I.B.S.) and Public Health (S.P., E.W.S., E.F.v.B.), Rotterdam, Erasmus MC, University Medical Center Rotterdam, Rotterdam, The Netherlands; and Department of Surgery-Traumatology (I.B.S.), Leiden University Medical Center, Leiden, The Netherlands.

Address for reprints: Akkie Nadir Ringburg, MD, Department of Surgery-Traumatology, Erasmus MC, University Medical Center Rotterdam, PO Box 2040, 3000 CA Rotterdam, The Netherlands; email: a.ringburg@erasmusmc.nl.

DOI: 10.1097/TA.0b013e3181f6bce8 prevention and trauma care, ${ }^{4}$ where the number of survivors of severe injury has rapidly risen, ${ }^{5}$ and therefore, the number of injury patients with long-term disability has increased. Furthermore, the measurement of disability is also important because many survivors of major trauma are young people, whose daily activities may greatly and sometimes permanently suffer from the consequences of trauma. ${ }^{4,6,7}$

In the long term, most survivors of major trauma still suffer from one or more permanent functional consequences. This has a negative impact on their health-related quality of life (HRQoL), which will remain far below the general population norms. ${ }^{7-9}$ This considerable burden of mortality and disability resulting from major trauma needs to be addressed.

Because of the considerable variety in functional consequences and recovery patterns of injuries, a uniform measurement of disability is a necessary and challenging task. However, it has been reported that in some specialties, there are numerous measures of quality of life and little standardization. ${ }^{8}$ This lack of uniform measurement also applies to functional outcome research in traumatology. Therefore, guidelines were recently published for the conduction of follow-up studies measuring injury-related disability. ${ }^{5}$ Herein, it is advised to use a combination of the EuroQol-5D (EQ-5D) and the Health Utilities Index (HUI) 3 in all studies on injury-related disability as common core of health outcome measures. The HUI (HUI2 and HUI3) and the EQ-5D are both generic measures and aim to cover the full spectrum of disease and disability. Generic instruments enable a uniform comparison of the functional consequences of different types of injuries among each other and with other health problems. However, this advised combination of generic health outcome measures has to date not been used in follow-up studies measuring disability after major trauma. Previous studies have either used other generic health outcome measures, such as the Quality of Well Being Scale, ${ }^{9}$ or were restricted to the use of EQ-5D. ${ }^{7,10}$ Measuring the consequences of major trauma with the HUI has not been conducted yet.

Well-standardized information on HRQoL of major trauma survivors may provide valuable insight into the frequency, nature, and predictors of residual disabilities and may lead to changes in (treatment) policies. The primary aim of this study was to assess the HRQoL of survivors of severe trauma 1 year after injury, specified 
according to all the separate dimensions of the EQ-5D and the HUI. Second, this study aimed to assess the factors that are predictive for poor functioning on those dimensions in the long term. In addition to the influence of sociodemographic, physical, and injury-related factors, we explored the effect of an advancement of prehospital trauma care, i.e., assistance of physician-staffed helicopter emergency medical services (HEMSs) at the scene of the incident.

\section{PATIENTS AND METHODS}

\section{Study Population and Design}

From January 2004 to July 2006, a prospective cohort study was conducted, including all consecutive patients with multiple injuries, with an $\mathrm{ISS}^{2} \geq 16$ and older than 14 years, who were presented to the emergency department of a Level I trauma center in a Dutch trauma region serving 4.9 million inhabitants. Patients who were pronounced dead on arrival at the scene of the incident were excluded. For the purpose of this study, a hospital trauma registry was started that documented the same variables as the major trauma outcome study database ${ }^{10}$ (i.e., age, Glasgow Coma Scale, ${ }^{11}$ Revised Trauma Score $[\mathrm{RTS}],{ }^{12}$ mechanism of injury, and injury specifics). Missing data were obtained from the original ambulance charts.

\section{Outcome Assessment}

The EQ-5D and HUIs (HUI2 and HUI3) were used as generic measures to analyze the HRQoL. The combination of the EQ-5D with the HUI3 is in accordance with international guidelines for conducting follow-up studies measuring injuryrelated disability. ${ }^{5}$ The EQ-5D and HUI are complementary with respect to the domains of the International Classification of Disabilities, Functioning and Health (ICF) stated by the World Health Organization. ${ }^{3}$

The generic EQ-5D classification of health ${ }^{13}$ covers the main health domains that are affected by injury, with particular focus on the participation level of the ICF. It allows for a proper description of a heterogeneous injury population and for discrimination among specific injuries. ${ }^{14}$ Moreover, the EQ-5D has been recommended for (economic) evaluation of trauma care at a consensus conference..$^{15}$ In this classification, health is defined along five dimensions: mobility, self-care, usual activities, pain/discomfort, and anxiety/depression. Each dimension has three levels: no problem, moderate problem, and severe problem. Subsequently, a domain-related scoring algorithm based on empiric valuations from the United Kingdom general population and subsequent statistical modeling is available by which each health status description can be expressed into a utility score (EQ-5D). ${ }^{16}$ This summary score ranges from 1 for perfect health to 0 for death and can be interpreted as a judgment on the relative desirability of a health status compared with perfect health.

The second part of the EQ-5D consists of a vertical Visual Analog Scale (VAS). This calibrated scale is marked 100 at the top, labeled "best imaginable health state" and 0 at the bottom, labeled "worst imaginable health state." 13

The HUI is a self-administered health status questionnaire consisting of 15 questions, which classifies respondents into either the HUI Mark 2 (HUI2) or the HUI Mark 3 (HUI3) health states. ${ }^{17}$ It covers the main health domains that are affected by injury, with particular focus on functional capacities. Results of the questionnaire are converted by an algorithm, into the levels of the complementary HUI 2 and HUI 3 classification system, ${ }^{18}$ to form 7- and 8-element health state vectors, respectively. From these vectors, single-attribute and overall health state utility scores are calculated using the respective HUI2 and HUI3 utility functions, ${ }^{19}$ with preferences derived from the general public.

At 12 months after trauma admission, all included patients received the written questionnaire by mail. In absence of response, patients received a phone call 1 month after the mailing to stimulate participation and increase the response rate.

\section{Sociodemographic, Injury, and Health Care-Related Characteristics}

From the literature, potential determinants of functional outcome were identified. ${ }^{20-22}$ These determinants of functional outcome were grouped into sociodemographic (age and gender, education level, household composition, and comorbidity), injury (ISS, RTS, and injury location), and health care-related characteristics (HEMS or emergency medical service [EMS]). Education was divided into primary school level or higher; household composition was divided into households existing of a single person or more persons; and comorbidity was divided into a group without a comorbidity, a group with only one comorbidity, and a group with two or more comorbidities. A comorbidity condition was defined as a previous disease at the time of trauma according to the patient or the family. The injury diagnosis was verified at the individual level with information from the hospital discharge register according to the "Abbreviated Injury Scale, 1990 Revision, Update 1998."23

Prehospital trauma care, the first link in the complex chain of trauma patient care, was upgraded in the Netherlands in 1995, when physician-staffed HEMSs were introduced in addition to nurse-staffed EMSs. For all major trauma patients in this study, the type of prehospital care (HEMS or EMS) provided was registered.

\section{Statistical Analysis}

The statistical analyses were performed using the Statistical Package for the Social Sciences version 12.0 (SPSS, Chicago, IL). A nonresponse analysis was performed by multivariable logistic regression. Age, gender, ISS, RTS, mechanism of injury, health status (EQ-5D summary score), and HEMS or EMS were tested as possible determinants of nonresponse. All significant variables $(p<0.05)$ were used to adjust for response bias. Subsequently, the respondents were weighted with the inverse probability of response resulting from the final model.

The Kolmogorov-Smirnov test was used to test for normality of the data. The Levene's test was applied to assess homogeneity of variance between groups. Because not all outcome measures showed normal distribution or equal variance, all items were regarded as nonparametric for the statistical analysis. Sociodemographic and injury-related charac- 
TABLE 1. Characteristics of the Study Population (Patients Surviving Major Trauma at 12-mo Follow-Up) by Gender

\begin{tabular}{|c|c|c|c|c|}
\hline & Overall & Male & Female & $p$ \\
\hline $\mathrm{N}$ & 246 & 162 & 84 & \\
\hline $\mathrm{Age}^{*}(\mathrm{yr})$ & $40(23-57)$ & $36(22-55)$ & $44(24-66)$ & $0.054^{\dagger}$ \\
\hline Blunt trauma ${ }^{*}$ & $238(97)$ & $158(98)$ & $80(95)$ & $0.450^{\S}$ \\
\hline Glasgow Coma Score* & $14(7-15)$ & $14(6-15)$ & $13(8-15)$ & $0.555^{\dagger}$ \\
\hline RTS* & $12(10-12)$ & $12(10-12)$ & $11(11-12)$ & $0.723^{\dagger}$ \\
\hline ISS* & $22(17-29)$ & $22(17-29)$ & $20(17-29)$ & $0.382^{\dagger}$ \\
\hline Prehospital intubation ${ }^{\ddagger}$ & $43(18)$ & $33(20)$ & $10(12)$ & $0.113^{\S}$ \\
\hline Comorbidity ${ }^{\ddagger}$ & $90(37)$ & $56(35)$ & $34(40)$ & $0.403^{\S}$ \\
\hline HEMS* & $101(41)$ & $70(43)$ & $31(37)$ & $0.412 *$ \\
\hline
\end{tabular}

* Data are displayed as median, with the first and third quartile given within brackets.

${ }^{\dagger}$ Mann-Whitney $U$ test.

\$ Patient numbers are displayed, with the percentages given within brackets.

$\S$ Fisher exact test.

teristics were tested as predictors of HRQoL in univariate and step-forward multivariable regression analyses. Differences regarding the mean utility scores were tested with a one-way analysis of variance, and $p$ values $<0.05$ were considered to indicate statistical significance.

\section{RESULTS}

During the study period of 30 months, 524 patients with multiple injuries (ISS $>15$ ) aged 14 years or older were admitted to the emergency department of the study hospital. Of these patients, $162(30.9 \%)$ died within 30 days after hospital admission, and the remaining 362 survivors were included in the prospective cohort study on HRQoL. Oneyear follow-up measurements of 246 patients (response rate, $68 \%$ ) were obtained (Table 1). Of the 116 patients who did not participate, 107 patients were untraceable, 1 patient could not be included because the patient had insufficient knowledge of the Dutch or English language to properly communicate about the investigation, and the remaining 8 patients were unwilling to participate.

\section{Patient Characteristics}

One hundred sixty-two patients $(66 \%)$ were male (Table 1). The median ISS of this study population was 22 , with a median age of 40 years. The majority of patients $(97 \%)$ sustained a blunt force trauma. Male survivors had a median age of 36 years and a median ISS of 22. Female patients were slightly older (median, 44 years) but with a similar ISS. There were no significant differences in trauma mechanism, disturbance of vital parameters, injury severity, or type of prehospital trauma care.

\section{Description of HRQoL 1 Year After Trauma}

The median EQ-5D utility score of 0.73 of the total population of major trauma patients was far below the Dutch general population norms (EQ-5D summary measure, 0.88; Table 2). ${ }^{24}$ A median EuroQol Visual Analog Scale (EQVAS) for the total population was calculated of 70. The median HUI2 and HUI3 scores for the total population were 0.81 and 0.65 , respectively. Gender and
TABLE 2. HRQoL of Severely Injured Patients at 12 mo After Trauma by Sociodemographic, Physical, and InjuryRelated Factors and Type of Prehospital Care

\begin{tabular}{|c|c|c|c|c|c|}
\hline \multirow[b]{2}{*}{ Determinants } & \multirow[b]{2}{*}{$\mathbf{N}$} & \multicolumn{4}{|c|}{ Median } \\
\hline & & EQ-5D & HUI2 & HUI3 & EQVAS \\
\hline Total study population & 246 & 0.73 & 0.81 & 0.65 & 70 \\
\hline \multicolumn{6}{|l|}{ Sociodemographic } \\
\hline \multicolumn{6}{|l|}{ Gender } \\
\hline Male & 162 & 0.80 & 0.83 & 0.68 & 74 \\
\hline Female & 84 & 0.69 & 0.77 & 0.50 & 68 \\
\hline \multicolumn{6}{|l|}{ Age (yr) } \\
\hline$<55$ & 176 & 0.76 & 0.82 & 0.68 & 72 \\
\hline$\geq 55$ & 70 & 0.69 & 0.78 & 0.49 & 69 \\
\hline \multicolumn{6}{|l|}{ Education } \\
\hline Primary & 45 & 0.73 & 0.78 & 0.44 & 70 \\
\hline Higher & 181 & 0.73 & 0.81 & 0.66 & 70 \\
\hline \multicolumn{6}{|c|}{ Household composition } \\
\hline Alone & 69 & 0.69 & 0.77 & 0.59 & 68 \\
\hline Not alone & 166 & 0.78 & 0.81 & 0.68 & 73 \\
\hline \multicolumn{6}{|l|}{ Physical } \\
\hline \multicolumn{6}{|l|}{ Comorbidity } \\
\hline None & 155 & 0.80 & 0.85 & 0.73 & 76 \\
\hline 1 & 67 & 0.60 & 0.76 & 0.47 & 66 \\
\hline$\geq 2$ & 23 & 0.64 & 0.61 & 0.31 & 55 \\
\hline \multicolumn{6}{|l|}{ Injury related } \\
\hline \multicolumn{6}{|l|}{ ISS } \\
\hline$<25$ & 145 & 078 & 0.81 & 0.68 & 73 \\
\hline$\geq 25$ & 101 & 0.72 & 0.80 & 0.59 & 70 \\
\hline \multicolumn{6}{|l|}{ Injury localization } \\
\hline \multicolumn{6}{|l|}{ Head } \\
\hline$<3$ & 65 & 0.69 & 0.82 & 0.66 & 68 \\
\hline$\geq 3$ & 181 & 0.76 & 0.80 & 0.64 & 73 \\
\hline \multicolumn{6}{|l|}{ Face } \\
\hline$<3$ & 240 & 0.73 & 0.80 & 0.65 & 70 \\
\hline$\geq 3$ & 6 & 0.72 & 0.73 & 0.48 & 76 \\
\hline \multicolumn{6}{|l|}{ Chest } \\
\hline$<3$ & 146 & 0.74 & 0.80 & 0.65 & 71 \\
\hline$\geq 3$ & 100 & 0.73 & 0.80 & 0.63 & 70 \\
\hline \multicolumn{6}{|l|}{ Abdomen } \\
\hline$<3$ & 213 & 0.73 & 0.81 & 0.65 & 70 \\
\hline$\geq 3$ & 33 & 0.76 & 0.82 & 0.65 & 70 \\
\hline \multicolumn{6}{|l|}{ Extremities } \\
\hline$<3$ & 185 & 0.76 & 0.81 & 0.67 & 71 \\
\hline$\geq 3$ & 61 & 0.69 & 0.78 & 0.55 & 70 \\
\hline \multicolumn{6}{|l|}{ Type of prehospital care } \\
\hline EMS & 145 & 0.73 & 0.83 & 0.66 & 70 \\
\hline HEMS & 101 & 0.76 & 0.80 & 0.64 & 70 \\
\hline
\end{tabular}

Utility scores of EQ-5D and HUIs (HUI2 and HUI3) were calculated as described in the Material and Methods. These scores range from 0 for death to 1 for perfect health; the EQVAS score ranges from 0 for the worst imaginable health state to 100 for the best imaginable health. Median scores are displayed. The population norm for the EQ-5D and EQVAS are 0.88 and 0.83 , respectively. The first row displays the median scores for the total study population. In all subsequent rows, utility and VAS scores of subgroups based on the determinants sociodemographic, physical and injury-related factors were compared. Values given in bold indicate a statistically significant difference in utility or VAS score between the indicated determinants (Mann-Whitney $U$ test, $p<0.05$ ). For comorbidity, pairwise comparison was made for all three groups. Statistical significance was reached when comparing absence of comorbidity vs. either one or multiple comorbidities. 
comorbidity were significantly and consistently associated with worse EQ-5D and HUI outcomes. Females reported worse 1-year follow-up health states compared with males. This difference was statistically significant for EQ-5D, HUI2, and EQVAS. In all generic measures used, one or more comorbidities were associated with worse HRQoL. The observed associations between the other included variables and HRQol were less consistent. Patients with a higher age (55 years or older) had significantly worse HUI3 and EQVAS scores. A household composition of more than one person was associated with a better reported HRQoL on the EQ utility score and EQVAS. Only the HUI3 showed an association of higher ISS $(\geq 25)$ with reduced HRQoL. Type of prehospital care has no influence on the reported quality of life in any of the EQ-5D or HUI2/HUI3 summary scores.

Of all major trauma patients, $18 \%$ had at least one functional limitation 1 year after injury, and $60 \%$ reported functional limitation on two or more domains using the EQ-5D. One year after trauma, the prevalence of physical and physiologic limitations for the total patient population was high on all dimensions of both EQ-5D (44\% for mobility, $19 \%$ for self-care, $53 \%$ for usual activities, $62 \%$ for pain and discomfort, and $41 \%$ for anxiety and depression; Fig. 1, A) and HUI3 (54\% for vision, $14 \%$ for hearing, $29 \%$ for speech, $29 \%$ for walking, $21 \%$ for dexterity, $65 \%$ for emotion, $55 \%$ for cognition, and $68 \%$ for pain; Fig. 1, B).

The presence of comorbidities on all the separate dimensions of EQ-5D and HUI3 was consistent and sig-
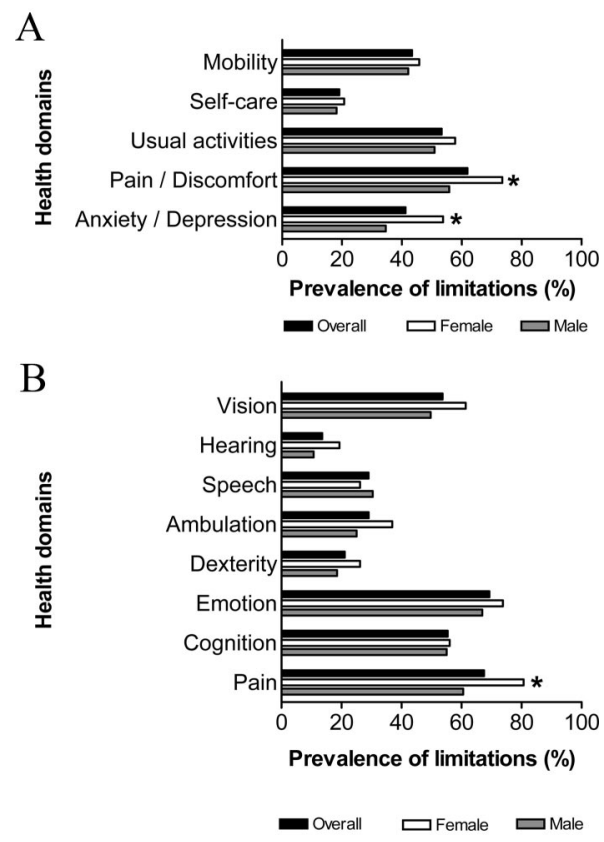

Figure 1. Prevalence of physical and physiological limitations (moderate or severe) of the EQ-5D $(A)$ and HUI3 $(B)$ health domains by gender. The percentage of patients with limitations in any of the health domain is shown. Differences between males and females were tested with the $\chi^{2}$ test. Asterisk indicates $p \leq 0.01$. nificant. On almost all dimensions of the EQ-5D (e.g., mobility, usual activities, pain/discomfort, and anxiety/ depression), the prevalence of limitations was higher in the presence of comorbidities. Comparable results were found using the HUI3, where the presence of comorbidities was related with functional limitations in almost all domains (vision, hearing, walking, emotion, cognition, and pain).

\section{Multivariable Analyses}

A multivariable regression analysis was conducted to further explore the influence of sociodemographic and injuryrelated factors and type of prehospital care on health status 1 year after trauma (Table 3). Posttrauma problems concerning anxiety/depression were significantly influenced by sociodemographic determinants and comorbidity. Females with a higher educational level and a household consisting of one person reported more problems concerning anxiety and depression. Females were also more likely to experience limitations because of pain and physical discomfort.

Absence of comorbidity was an independent predictor for less mobility-related limitations (odds ratio [OR] = $0.5)$, limitations for usual activities $(\mathrm{OR}=0.4)$, pain/discomfort $(\mathrm{OR}=0.2)$, and anxiety/depression $(\mathrm{OR}=0.3)$. Patients with a higher ISS $(\geq 25)$ were more likely to report limitations concerning mobility, self-care, and usual activities. Patients who sustained severe chest injuries showed less problems on several health domains, compared with patients with severe injuries of other body regions. This association was only significant for less limitation in self-care. As expected, severe injuries to the extremities were significant independent predictors of limitations in mobility.

Comparable results as shown for the EQ-5D were found in a separate multivariable regression analysis with the HUI as outcome measure (data not shown). In this analysis too, the absence of comorbidity was a significant independent predictor for fewer limitations concerning the HUI dimensions: walking $(\mathrm{OR}=0.3)$, emotion $(\mathrm{OR}=0.5)$, cognition $(\mathrm{OR}=0.3)$, and pain $(\mathrm{OR}=0.4)$. Comparable with the results found with the EQ-5D, the HUI showed that females were more likely to experience problems concerning pain compared with men $(\mathrm{OR}=0.4)$. Patients with a higher ISS $(\geq 25)$ were more likely to report limitations concerning walking $(\mathrm{OR}=2.6)$ and dexterity $(\mathrm{OR}=2.9)$. As expected, severe injuries to the extremities were independent predictors of dexterity $(\mathrm{OR}=4.1)$. No significant differences on any of the separate functional outcome dimensions of the HUI were found between patients assisted by HEMS or EMS. The type of prehospital care showed no differences on any of the separate dimensions of the EQ-5D. In comparing HEMS with EMS, the ORs were ranging from $0.6(95 \%$ confidence interval $=0.2-1.3)$ for the dimension self-care to $1.8(95 \%$ confidence interval $=$ $0.9-3.6)$ for the dimension anxiety/depression.

\section{DISCUSSION}

One year after trauma, the average day-to-day function of major trauma patients has not returned to normal in this study population. HRQoL, as measured by the summary 
TABLE 3. ORs of Determinants of Limitations of Functional Outcome After Major Trauma Assessed by Multivariable Logistic Regression Analyses

\begin{tabular}{|c|c|c|c|c|c|}
\hline & EQ-1 Mobility & EQ-2 Self-Care & EQ-3 Usual Activities & EQ-4 Pain/Discomfort & EQ-5 Anxiety/Depression \\
\hline \multicolumn{6}{|l|}{ Sociodemographic } \\
\hline Male & $1.0(0.5-1.9)$ & $0.9(0.4-1.9)$ & $0.8(0.5-1.6)$ & $0.4(0.2-0.8)^{*}$ & $0.4(0.2-0.8)^{\dagger}$ \\
\hline Age $<55$ yr & $0.7(0.4-1.5)$ & $0.8(0.3-1.9)$ & $1.0(0.5-2.1)$ & $1.5(0.7-3.1)$ & $1.3(0.6-2.6)$ \\
\hline Primary education & $2.0(1.0-4.2)$ & $1.5(0.6-3.7)$ & $1.2(0.6-2.6)$ & $0.7(0.3-1.5)$ & $0.4(0.2-1.0)^{*}$ \\
\hline Living alone & $1.6(0.8-3.0)$ & $1.1(0.5-2.5)$ & $1.7(0.9-3.3)$ & $1.7(0.8-3.5)$ & $2.3(1.2-4.6)^{*}$ \\
\hline \multicolumn{6}{|l|}{ Physical } \\
\hline No comorbidity & $0.5(0.2-0.8)^{*}$ & $0.5(0.2-1.2)$ & $0.4(0.2-0.8)^{\dagger}$ & $0.2(0.1-0.5)^{\dagger}$ & $0.3(0.1-0.5)^{\dagger}$ \\
\hline \multicolumn{6}{|l|}{ Injury related } \\
\hline ISS value $\geq 25$ & $2.3(1.1-4.9)^{*}$ & $5.2(2.1-12.8)^{\dagger}$ & $2.6(1.2-5.6)^{*}$ & $0.9(0.4-1.9)$ & $1.0(0.5-2.1)$ \\
\hline \multicolumn{6}{|l|}{ Injury localization } \\
\hline Head $\geq 3$ & $0.8(0.3-2.0)$ & $0.4(0.1-1.1)$ & $0.6(0.2-1.4)$ & $0.4(0.1-1.1)$ & $1.2(0.5-3.1)$ \\
\hline Face $\geq 3$ & $0.9(0.1-5.5)$ & 0 & $4.7(0.5-44.2)$ & $1.9(0.3-12.9)$ & $1.7(0.3-10.4)$ \\
\hline Chest $\geq 3$ & $0.6(0.3-1.3)$ & $0.3(0.1-0.9)^{*}$ & $0.7(0.3-1.5)$ & $1.2(0.5-2.8)$ & $0.9(0.4-1.9)$ \\
\hline Abdomen $\geq 3$ & $0.8(0.3-2.2)$ & $1.0(0.3-3.0)$ & $0.7(0.3-1.9)$ & $0.8(0.3-2.4)$ & $1.5(0.5-4.3)$ \\
\hline Extremities $\geq 3$ & $2.3(1.0-4.9)^{*}$ & $1.6(0.6-4.0)$ & $0.8(0.4-1.8)$ & $1.6(0.7-3.9)$ & $0.9(0.4-2.0)$ \\
\hline HEMS & $0.9(0.5-1.6)$ & $0.6(0.2-1.3)$ & $0.8(0.4-1.5)$ & $1.4(0.7-2.8)$ & $1.8(0.9-3.6)$ \\
\hline \multicolumn{6}{|r|}{ Sof the FO-5D (FO- 1 to $F O-5$ ) } \\
\hline
\end{tabular}

scores of both the EQ-5D and HUI, remained far below general population norms. Of all major trauma patients, $18 \%$ had at least one functional limitation 1 year after injury, and $60 \%$ reported functional limitation on two or more domains using the EQ-5D. The prevalence of specific limitations in this population was very high, with $40 \%$ to $70 \%$ of patients still suffering from problems with a specific dimension, such as mobility (44\%), usual activities $(53 \%)$, pain $(62 \%-68 \%)$, anxiety/depression (41\%), emotion (65\%), and cognition (55\%) after 1 year.

Female gender and comorbidity were predictive for long-term disability. Because this was the first study applying the HUI, we could add prevalences of problems among major trauma patients with dexterity $(21 \%)$, cognition (55\%), and emotion $(65 \%)$ to the literature.

In this study, the effect of an advancement of prehospital trauma care, i.e., assistance of physician-staffed HEMSs at the scene of the incident was also explored. No difference in outcomes between patients receiving more or less advanced prehospital trauma care has been found. HRQoL at 1 year after major trauma was far more influenced by personal factors than by the level of prehospital care, as reflected by the significant and consistent negative effects of female gender and comorbidity on the (dimensions of the) EQ-5D and HUI.

Our main findings, as summarized above, are based on a prospective cohort study of severely injured survivors in a Dutch trauma region. This study was designed according to international guidelines for the conduction of follow-up studies measuring injury-related disability. ${ }^{5}$ First, in this study, the internationally accepted case definition for major trauma (ISS $>15^{2}$ ) was used, and no previous exclusions of patients based on social characteristics (e.g., language and ethnicity) were made. As recommended, HRQoL was measured with EQ-5D and HUI to cover all health dimensions of the ICF that are relevant for patients with (major) trauma. In previous studies, ${ }^{7,25}$ determinants of long-term functional consequences of major trauma have demonstrated good performance of EQ-5D in major trauma survivors, in terms of discriminative power and sensitivity to change. Nevertheless, some limitations of EQ-5D were identified (e.g., lacking information on dexterity and cognition) that have been addressed in this study by additionally applying the HUI. The validity of our descriptive results is supported by the consistency of results on the EQ-5D and HUI, respectively. The prevalence of pain (i.e., the single dimension with full overlap between both measures) was comparably high on both the EQ-5D (62\%) and the HUI (68\%). High prevalences of limitations on all health domains were consistently found on both measures.

Because well-validated instruments were used, the reported high prevalence of health-related limitations in this study is a good reflection of the health situation of major trauma patients after 1 year. This provides support for our finding that 1 year after trauma, generic average HRQoL is not different in patients with HEMS or EMS assistance and is far more influenced by personal factors (as reflected by the significant and consistent negative effects of female gender and comorbidity) than by the level of prehospital care. This negative influence of comorbidity and female gender is consistent with previous reports. Numerous investigators have previously reported that comorbidity is an important independent predictor of worse health outcomes after major trauma. ${ }^{22,25-28}$ Our results show that comorbidity is a predic- 
tor of adverse functional outcome after major trauma. Therefore, as suggested by previous authors, comorbidity should be included in the standardized set of determinants in quality of life studies. ${ }^{25}$

The influence of the female gender as an independent predictor of worse functional outcome after major trauma has also been reported in different studies. ${ }^{7,14,29,30}$ Vles et al. ${ }^{7}$ hypothesized that the relation between adverse outcomes and the female gender could be related to physiologic, psychologic, and social differences between males and females.

We found that females experience worse generic HRQoL in the long term, mainly because of significantly more problems on psychologic dimensions. Comparable with previous studies, no association was found with extremity injuries and problems in self-care. ${ }^{7,25}$

At 1 year after trauma, both in comparing the crude data and after adjustment for injury severity and other confounders (including age and comorbidity), no statistical significant differences in HRQoL between HEMS- and EMSassisted patients were found. This indicates that HEMS assistance leads neither to a shift from mortality to injuryrelated morbidity and disability nor to improved functional outcome in the long term. This result is consistent with the small amount of previous studies on this topic. Oppe and De Charro $^{31}$ found comparable EQ-5D summary scores of 0.67 and 0.71 for the Amsterdam population at 9 and 15 months, respectively. Overall, they found that the quality of life was lower for the HEMS population compared with the EMS group. However, after correcting for injury severity, no differences in functional outcome remained. Similar results were found in the United Kingdom. Six months after trauma, no differences in health status, measured by the Nottingham Health Profile, were found between EMS- and HEMSassisted patients. ${ }^{32}$ In addition, a small study performed in Finland using the SF-36 quality of life questionnaire could not demonstrate an improved HRQoL by a physician-staffed HEMS assistance. ${ }^{33}$

More efforts are needed to improve the HRQoL of major trauma patients. The prevalence of reported limitations after major trauma is high, and advanced prehospital trauma care alone seems not enough to achieve more acceptable outcomes.

\section{CONCLUSIONS}

Functional outcome and quality of life of survivors of severe injury have not returned to normal 1 year after trauma. The prevalence of specific limitations in this population is very high $(40-70 \%)$. Female gender and comorbidity are predictors of long-term disability. HRQoL at 1 year after major trauma was far more influenced by personal factors than by the level of prehospital care.

\section{ACKNOWLEDGMENTS}

We thank Ms. Diana van Emmerik and Ms. Tamara Meulman for their assistance in data collection.

\section{REFERENCES}

1. Krug EG, Sharma GK, Lozano R. The global burden of injuries. Am $J$ Public Health. 2000;90:523-526.

2. Baker SP, O'Neill B, Haddon W Jr, Long WB. The injury severity score: a method for describing patients with multiple injuries and evaluating emergency care. J Trauma. 1974;14:187-196.

3. World Health Organization. International Classification of Functioning, Dissabilities and Health (ICF). Geneva, Switzerland: World Health Organization; 2001.

4. Segui-Gomez M, MacKenzie EJ. Measuring the public health impact of injuries. Epidemiol Rev. 2003;25:3-19.

5. van Beeck EF, Larsen CF, Lyons RA, Meerding WJ, Mulder S, EssinkBot ML. Guidelines for the conduction of follow-up studies measuring injury-related disability. $J$ Trauma. 2007;62:534-550.

6. Barker M, Power C, Roberts I. Injuries and the risk of disability in teenagers and young adults. Arch Dis Child. 1996;75:156-158.

7. Vles WJ, Steyerberg EW, Essink-Bot ML, van Beeck EF, Meeuwis JD, Leenen LP. Prevalence and determinants of disabilities and return to work after major trauma. J Trauma. 2005;58:126-135.

8. Holbrook TL, Anderson JP, Sieber WJ, Browner D, Hoyt DB. Outcome after major trauma: discharge and 6-month follow-up results from the Trauma Recovery Project. J Trauma. 1998;45:315-323; discussion 323324.

9. Holtslag HR, van Beeck EF, Lichtveld RA, Leenen LP, Lindeman E, van der Werken C. Individual and population burdens of major trauma in the Netherlands. Bull World Health Organ. 2008;86:111117.

10. Champion HR, Copes WS, Sacco WJ, et al. The Major Trauma Outcome Study: establishing national norms for trauma care. J Trauma. 1990;30: $1356-1365$.

11. Teasdale G, Jennett B. Assessment of coma and impaired consciousness. A practical scale. Lancet. 1974;2:81-84.

12. Champion HR, Sacco WJ, Copes WS, Gann DS, Gennarelli TA, Flanagan ME. A revision of the Trauma Score. J Trauma. 1989;29: 623-629.

13. EuroQol - a new facility for the measurement of health-related quality of life. The EuroQol Group. Health Policy. 1990;16:199-208.

14. Meerding WJ, Looman CW, Essink-Bot ML, Toet H, Mulder S, van Beeck EF. Distribution and determinants of health and work status in a comprehensive population of injury patients. J Trauma. 2004;56:150161.

15. Bouillon B, Kreder HJ, Eypasch E, et al. Quality of life in patients with multiple injuries - basic issues, assessment, and recommendations. Restor Neurol Neurosci. 2002;20:125-134.

16. Dolan P. Modeling valuations for EuroQol health states. Med Care. 1997;35:1095-1108.

17. Furlong W, Feeny D, Torrance GW. Health Utilities Index (HUIC) Procedures Manual: Algorithm for Determining HUI Mark 2 (HUI2)/ Mark 3 (HUI3) Health Status Classification Levels, Health States, Single-Attribute Utility Scores and Overall Health-Related Quality of Life Utility Scores, From 15-Item Self-Complete Health States Questionnaires. Hamilton, Ontario, Canada: Health Utilities Inc.; 2000.

18. Feeny D, Furlong W, Boyle M, Torrance GW. Multi-attribute health status classification systems. Health Utilities Index. Pharmacoeconomics. 1995;7:490-502.

19. Furlong W, Feeny D, Torrance GW, et al. Multiplicative, MultiAttribute Utility Function for the Health Utilities Index Mark 3 (HUI3) System: A Technical Report. Hamilton, Ontario, Canada: Centre for health Economics and Policy Analysis, McMaster University; 1998:98-111.

20. Holbrook TL, Anderson JP, Sieber WJ, Browner D, Hoyt DB. Outcome after major trauma: 12-month and 18-month follow-up results from the Trauma Recovery Project. J Trauma. 1999;46:765-771; discussion 771763.

21. Holbrook TL, Hoyt DB. The impact of major trauma: quality-of-life outcomes are worse in women than in men, independent of mechanism and injury severity. J Trauma. 2004;56:284-290.

22. Polinder S, van Beeck EF, Essink-Bot ML, et al. Functional outcome at 2.5, 5, 9, and 24 months after injury in the Netherlands. $J$ Trauma. 2007;62:133-141. 
23. Association for the Advancement of Automotive Medicine. The Abbreviated Injury Scale, 1990 Revision, Update 98. Des Plaines, IL: Association for the Advancement of Automotive Medicine; 1998.

24. Hoeymans N, van Lindert H, Westert GP. The health status of the Dutch population as assessed by the EQ-6D. Qual Life Res. 2005;14:655-663.

25. Holtslag HR, van Beeck EF, Lindeman E, Leenen LP. Determinants of long-term functional consequences after major trauma. J Trauma. 2007; 62:919-927.

26. Bergeron E, Lavoie A, Moore L, Clas D, Rossignol M. Comorbidity and age are both independent predictors of length of hospitalization in trauma patients. Can J Surg. 2005;48:361-366.

27. Poses RM, McClish DK, Smith WR, Bekes C, Scott WE. Prediction of survival of critically ill patients by admission comorbidity. J Clin Epidemiol. 1996;49:743-747.

28. Wardle TD. Co-morbid factors in trauma patients. Br Med Bull. 1999 $55: 744-756$
29. Holbrook TL, Hoyt DB, Anderson JP. The impact of major in-hospital complications on functional outcome and quality of life after trauma. J Trauma. 2001;50:91-95.

30. Polinder S, Meerding WJ, Toet H, Mulder S, Essink-Bot ML, van Beeck EF. Prevalence and prognostic factors of disability after childhood injury. Pediatrics. 2005;116:e810-e817.

31. Oppe S, De Charro FT. The effect of medical care by a helicopter trauma team on the probability of survival and the quality of life of hospitalised victims. Accid Anal Prev. 2001;33:129-138.

32. Snooks HA, Nicholl JP, Brazier JE, Lees-Mlanga S. The costs and benefits of helicopter emergency ambulance services in England and Wales. J Public Health Med. 1996;18:67-77.

33. Lirola TT, Laaksonen MI, Vahlberg TJ, Pälve HK. Effect of physician-staffed helicopter emergency medical service on blunt trauma patient survival and prehospital care. Eur J Emerg Med. 2006;13: 335-339. 